\title{
Preparation and Manipulation of Olfactory Epithelium Explant Cultures for Measurement of the Mechanical Tension of Individual Axons Using the Biomembrane Force Probe \\ Coralie Fouquet and Alain Trembleau*
}

Sorbonne Université, CNRS, INSERM, Institut de Biologie Paris Seine, Laboratoire Neuroscience Paris Seine, F-75005 Paris, France

*For correspondence: alain.trembleau@sorbonne-universite.fr

\begin{abstract}
[Abstract] In this paper, we describe a protocol allowing measurement of the mechanical tension of individual axons grown ex vivo from neural tissue explants. This protocol was developed with primary cultures of olfactory epithelium explants from embryonic (E13.5) mice. It includes a detailed description of explant dissection and culture, as well as the main steps of the procedure for axon tension measurement using the previously established Biomembrane Force Probe.
\end{abstract}

Keywords: Axon, Neuromechanics, Explant culture, Olfactory sensory neuron, Mechanical tension, Biomembrane force probe

[Background] The development of neural networks involves the growth and navigation of axons toward their target. It has recently been shown that neural network development depends not only on molecular cues acting on the developing axon, but also on extrinsic and intrinsic mechanical forces (Reviewed in Franze et al., 2013, Franze, 2013, Gangatharan et al., 2018). In the course of a study analyzing the mechanisms of fasciculation/defasciculation of olfactory sensory axons, we discovered that the mechanical tension of axons plays a critical role in regulating these processes (Šmít et al., 2017a). The mechanical tension of PC-12 axons or dorsal root ganglia neurites, as well as motor neuron axons in Drosophila embryo, had previously been measured using calibrated needles or microelectromechanical systems (Dennerll et al., 1988 and 1989; Rajagopalan et al., 2010). These approaches, however, appeared inappropriate for olfactory axons characterized by a very small diameter $(200 \mathrm{~nm})$, mostly because physical contact of the manipulator with the substrate would be inevitable, and would lead to inaccurate force measurements. To assess the mechanical tension of these thin axons, we developed a new protocol using the Biomembrane Force Probe (BFP) technique, in which a red blood cell is used as a force transducer (Evans et al., 1995; Gourier et al., 2008), adapted here for the first time to axon manipulations ex vivo. In our protocol, live axons are biotinylated, then streptavidin-coated glass microbeads ( $3 \mu \mathrm{m}$ diameter) are attached to the biotinylated axons; these beads are then manipulated with a biotinylated red blood cell held by an aspirating micropipette (Šmít et al., 2017a and 2017b). The force with which the bead is manipulated can be calculated by measuring the deformation of the red blood cell, using our recently developed software (Šmít et al., 2017b). 


\section{Materials and Reagents}

A. Materials

Note: All material and reagents used for the explant cultures must be sterile. Tools used for explant culture are autoclaved.

1. 26 G $1 \frac{1}{2}$ Needle (TERUMO, Dutscher, catalog number: 50101)

2. $1.5 \mathrm{ml}$ Eppendorf tube

3. 4 well plates (to store tissue during explants preparation); un-treated 4-well IVF Dish (Thermo Fisher Scientific, Nunc ${ }^{\top \mathrm{M}}$, catalog number: 179830)

4. $10 \mathrm{~cm}$ diameter plastic Petri dish (to collect embryos)

5. 6 (to 10 ) $\mathrm{cm}$ diameter Glass Petri dishes (2 units-for epithelium dissection and explant cutting)

6. $50 \mathrm{~mm}$ diameter IBIDI video dishes u-Dish $50 \mathrm{~mm}$, low ibiTreat: $\varnothing 50 \mathrm{~mm}$, low wall ( $3 \mathrm{ml}$ volume), \#1.5 polymer coverslip, tissue culture treated, sterilized (Biovalley, catalog number: 81136)

7. Sterile pipette tips

8. $10 \mathrm{ml}$ pipettes

9. Sterile $15 \mathrm{ml}$ and $50 \mathrm{ml}$ tubes (Falcon, Dutscher, catalog numbers: 352096; 352070)

10. $0.2 \mu \mathrm{m}$ filters $+20 \mathrm{ml}$ syringes to filter the media

11. Borosilicate glass capillaries GC100-15 (Harvard apparatus Ltd., Kent, UK)

B. Animals

Embryonic day 13.5 (E13.5) embryos were obtained from pregnant mice. Swiss or C57BI6/J female mice were used, and gave similar results.

C. Reagents

Note: Work under sterile conditions to prepare all reagents, aliquots and recipes. Aliquots and recipes are prepared for 3 plates.

1. Ice

2. Sterile $1 \times \mathrm{PBS}\left(-\mathrm{CaCl}_{2},-\mathrm{MgCl}_{2}\right)$ (Gibco, catalog number: $\left.14190[100 \mathrm{ml}]\right)$, store at $4{ }^{\circ} \mathrm{C}$

3. Sterile ultrapure $\mathrm{H}_{2} \mathrm{O}$

4. Leibovitz's L-15 (w/o Phenol Red) (Gibco, catalog number: 21083-027) (500 ml), Prepare working aliquots of $47.5 \mathrm{ml} \mathrm{L-15}$ in $50 \mathrm{ml}$ tubes, store at $4{ }^{\circ} \mathrm{C}$

5. Poly-L-Lysine (PLL) (Sigma, catalog number: P1524), resuspend at $2 \mathrm{mg} / \mathrm{ml}$ in $\mathrm{H}_{2} \mathrm{O}$ and prepare working aliquots of $145 \mu \mathrm{l}$ in $1.5 \mathrm{ml}$ microtubes. store at $-20^{\circ} \mathrm{C}$

6. Poly-D-Lysine (alternative, see Notes) (Sigma, catalog number: P6407), resuspend at $1 \mathrm{mg} / \mathrm{ml}$ in $\mathrm{H}_{2} \mathrm{O}$ and prepare working aliquots of $15 \mu \mathrm{l}$ in $1.5 \mathrm{ml}$ microtubes, store at $-20{ }^{\circ} \mathrm{C}$

7. Laminin (Sigma, catalog number: L2020) $(1 \mathrm{mg} / \mathrm{ml})$, prepare working aliquots of $20 \mu \mathrm{l}$ in $1.5 \mathrm{ml}$ microtube, store at $-20^{\circ} \mathrm{C}$ 
8. N2 supplement (Gibco, catalog number: 17502-048), prepare working aliquots of $200 \mu \mathrm{l}$, store at $-20^{\circ} \mathrm{C}$

9. Fetal Bovine Serum (FBS) (Gibco, catalog number: 10270), store $5 \mathrm{ml}$ heat inactivated aliquots (see Recipes, working aliquots 1 ) at $-20^{\circ} \mathrm{C}$

10. D-(+)-Glucose 30\% (Sigma, catalog number: G-8769 [powder]) (see Recipes, working aliquots 2)

11. Bovine serum albumin (BSA) (Sigma, catalog number: A-4161 [powder]), store at $4{ }^{\circ} \mathrm{C}$ (see Recipes, working aliquots 3 )

12. Gentamycin, $10 \mathrm{mg} / \mathrm{ml}$ (Sigma, catalog number: G-1272), store at $4{ }^{\circ} \mathrm{C}$

13. DMEM/F-12 (Glutamax supplement) (Gibco, catalog number: 31331-028 [500 ml]), store at $4{ }^{\circ} \mathrm{C}$

14. $0.25 \%$ Trypsin (Gibco, catalog number: 25050 ), prepare working aliquots of $200 \mu \mathrm{l}$ aliquots in $1.5 \mathrm{ml}$ microtubes, store at $-20^{\circ} \mathrm{C}$

15. 4x USP Pancreatin (Gibco, catalog number: 02-0036DG), prepare working aliquots of $200 \mu \mathrm{l}$ in $1.5 \mathrm{ml}$ microtubes, store at $-20^{\circ} \mathrm{C}$

16. Ascorbic acid $5 \mathrm{mg} / \mathrm{ml}$ (Sigma, catalog number: A-4403), store at $4{ }^{\circ} \mathrm{C}$

17. Reagents for cell surface biotinylation: EZ-Link Sulfo-NHS-SS-Biotin, No-Weigh Format, $8 \times 1$ mg microtubes (Thermo Fisher Scientific, catalog number: 21328), Waltham, Massachusets, store at $-20^{\circ} \mathrm{C}$

18. Streptavidin-coated beads (Gourier et al., 2008) (alternative, see Notes)

19. (Optional) HEPES $1 \mathrm{M}$ (Thermo Fisher Scientific, Gibco, catalog number: 15630080) (see Notes)

20. Reagent for red blood cell biotinylation: PEG-biotin polymer NHS-PEG 3400-biotin (Interchim, Montluçon, France), store at $-20^{\circ} \mathrm{C}$

21. $\mathrm{NaHCO}_{3}$

22. $\mathrm{Na}_{2} \mathrm{CO}_{3}$

23. $\mathrm{NaCl}$

24. Tris base

25. $\mathrm{KH}_{2} \mathrm{PO}_{4}$

26. $\mathrm{K}_{2} \mathrm{HPO}_{4}$

27. Paraformaldehyde powder (Sigma, catalog number: P6148), used for fixation (Prepare a $4 \%$ solution in $1 \times$ PBS)

28. Triton $^{\mathrm{TM}} \mathrm{X}-100$ (Sigma), used for permeabilization step

29. Normal Donkey Serum (NDS, Sigma, catalog number: D9663-10ML) for blocking step

30. Rat $\alpha-N c a m$ (Millipore, catalog number: MAB310) coupled to Donkey $\alpha$-Rat-Cy3 (Jackson Immunoresearch, catalog number: 712-166-153), used for labeling OSNs axons

31. Streptavidin-Fitc (FluoProbes, catalog number: FP-20937A), used for labeling biotin attached to axons

32. Hoechst (Sigma, catalog number: H33258), used for labeling OSNs nuclei 
33. Mowiol ${ }^{\circledR}$ 4-88 (Sigma, catalog number: 81381) (the mounting medium used)

34. Working aliquot 1 (see Recipes)

35. Working aliquot 2 (see Recipes)

36. Working aliquot 3 (see Recipes)

37. Solution A (see Recipes)

38. Solution $B$ (see Recipes)

39. Solution $C$ (see Recipes)

40. Solution D (see Recipes)

41. Solution E (see Recipes)

42. Solution $F$ (see Recipes)

43. Biotinylated Red Blood Cells (RBCs) (see Recipes)

\section{Equipment}

1. Micropipettes

2. P-2000 Pipette puller (Sutter Instruments, Novato California, USA)

3. Perforated spoon (Moria MC17) (FST, catalog number: 10370-17) or curved forceps Moria 9987 (FST, catalog number: 11399-87) (used to transfer embryo)

4. Forceps: Moria MC40 Forceps-Straight/11cm (FST, 11370-40)

5. Scissors: Moria 9600 Spring Scissors-Straight (FST, 15396-00)

6. Scalpel: Standard incision scalpel, $45^{\circ}$, Aluminum handle, $n^{\circ} 745$, FEATHER (pfm medical, catalog number: 200300745)

7. Horizontal laminar flow hood (Techgen international, OSN5)

8. $\mathrm{CO}_{2}$ Incubator (Fisher Bioblock Scientific, Thermo Scientific Forma 371 Steri Cycle $\mathrm{CO}_{2}$ Incubator)

9. Water bath (Fisher Scientific, Isotemp 105) (to pre-heat medium and reagents at $37^{\circ} \mathrm{C}$ )

10. Stereomicroscope (Leica M80), achromatic objective $0.63 \mathrm{x}$ for height/length working distance (Leica 10450160) with an illuminated base (Leica 10450123); additional illumination sources: Leica CLS 150X/MED and photonic combination lightguides Leica 30140001

11. Vortex

12. Micro-centrifuge

13. Homemade microforge

14. DMIRB inverted microscope (Leica, Solms, Germany) equipped with a $63 x$ Leica objective, as well as a CCD camera (JAI, Yokohama, Japan), a digitally controlled heating box (Life Imaging Services $\mathrm{GmbH}$, Reinach, Switzerland), and with a micropipette holder coupled to a linear piezoelectric translator (Physik Instrumente, Karlsruhe, Germany) (Gourier et al., 2008)

15. Homemade hydraulic/pneumatic system connected to the micropipette, that provides the necessary control of the aspiration force applied to the probe (Gourier et al., 2008) 


\section{Procedure}

A. Explant culture

The day before explant preparation

1. Write all necessary information on each IBIDI video dish at this step (date, sample, condition test). Protect the bottom of the glass dishes from scratches by using a plastic tray. Typically 3 IBIDI video dishes are needed per experiment.

2. Under the hood (sterile conditions), coat the glass coverslips as follows (only the center of the coverslip needs to be coated):

a. Dilute a Poly-L-Lysine working aliquot $(2 \mathrm{mg} / \mathrm{ml})$ to $200 \mu \mathrm{g} / \mathrm{ml}$ in $1 \times \mathrm{PBS}\left(-\mathrm{CaCl}_{2},-\mathrm{MgCl}_{2}\right)$, e.g., for PLL coating of 3 dishes, add 9 volumes $(1,305 \mu \mathrm{l})$ of $1 \times \mathrm{PBS}\left(-\mathrm{CaCl}_{2},-\mathrm{MgCl}_{2}\right)$ in a $145 \mu \mathrm{l} \mathrm{PLL} 2 \mathrm{mg} / \mathrm{ml}$ aliquot to obtain $200 \mu \mathrm{g} / \mathrm{ml} \mathrm{PLL}$, and pipet $400 \mu \mathrm{l} \mathrm{PLL}$ in the center of each well. Flatten the drop with the tip (to enlarge it) without touching the glass. Don't let the drop touch the plastic part of the plate; it will form a meniscus and the central part of the coverslip won't be correctly coated (Figure 1B').

b. Carefully transfer the dishes in the incubator and incubate overnight at $37^{\circ} \mathrm{C}, 5 \% \mathrm{CO}_{2}$.

\section{The day of explant preparation}

1. Equilibrate all necessary reagents at $4{ }^{\circ} \mathrm{C}$ on ice (1x PBS $\left[-\mathrm{CaCl}_{2},-\mathrm{MgCl}_{2}\right]$, Laminin, L-15, FBS aliquot for Solution 1 preparation, trypsin and pancreatin aliquots, DMEM/F-12 and small aliquots for culture medium preparation-Solution 2).

2. Remove the dishes from the incubator. Under the hood, wash the PLL three times for 5 min each with sterile $\mathrm{H}_{2} \mathrm{O}$. Let the coverslip dry completely under the hood (with the cover removed).

3. Dilute Laminin (stock: $1 \mathrm{mg} / \mathrm{ml}$ ) to $20 \mu \mathrm{g} / \mathrm{ml}$ in $1 \times$ PBS $\left(-\mathrm{CaCl}_{2},-\mathrm{MgCl}_{2}\right.$ ), e.g., for 3 dishes Laminin coating, add $980 \mu \mathrm{l}$ x PBS (- $\mathrm{CaCl}_{2},-\mathrm{MgCl}_{2}$ ) to a $20 \mu \mathrm{l}$ Laminin $1 \mathrm{mg} / \mathrm{ml}$ aliquot and apply $300 \mu \mathrm{l}$ of this diluted Laminin solution to the center of each well. It will naturally take the place of the PLL coating.

4. Incubate the dishes for at least $3 \mathrm{~h}$ at $37^{\circ} \mathrm{C}, 5 \% \mathrm{CO}_{2}$.

Note: The next steps can be done during this coating.

5. Prepare the L-15; 5\% FBS (Solution A) and store it on ice.

6. Prepare the culture medium (Solution B) and store it on ice until the explants are ready.

7. Set up the material under the hood (stereomicroscope, glass dish containing cold L-15, forceps, scalpels, tips and pipettes (Figures 1B and $1 \mathrm{C}$ ).

8. Embryo collection

Note: All animal procedures must be approved by an Ethics Committee (for our own experiments, the Ile-de-France Ethics Committee).

For each experiment, we typically prepare 20 to 30 explants from a total of 3 to 4 embryos 
extracted from a pregnant female at embryonic day 13.5 (e.g., 5-6 explants per dish, around 20 explants necessary for 3 dishes).

a. Prepare 1 plastic Petri dish containing 1x PBS to collect the embryos, and keep it on ice.

b. Euthanize the pregnant female in accordance with the approved protocol validated by the Ethics Committee. In our case, pregnant female Swiss mice were euthanized by cervical elongation at embryonic day 13.5 (E13.5).

c. Extract the uterine horns containing the embryos from the pregnant mouse (Figure 1A), and collect them in the PBS-containing plastic Petri dish.

d. Proceed now with all the subsequent steps under the hood.

e. Extract embryos from the uterus and the chorion, respectively. Keep them on ice.

f. Transfer the embryos in glass Petri dish containing cold L-15 to the stereomicroscope.
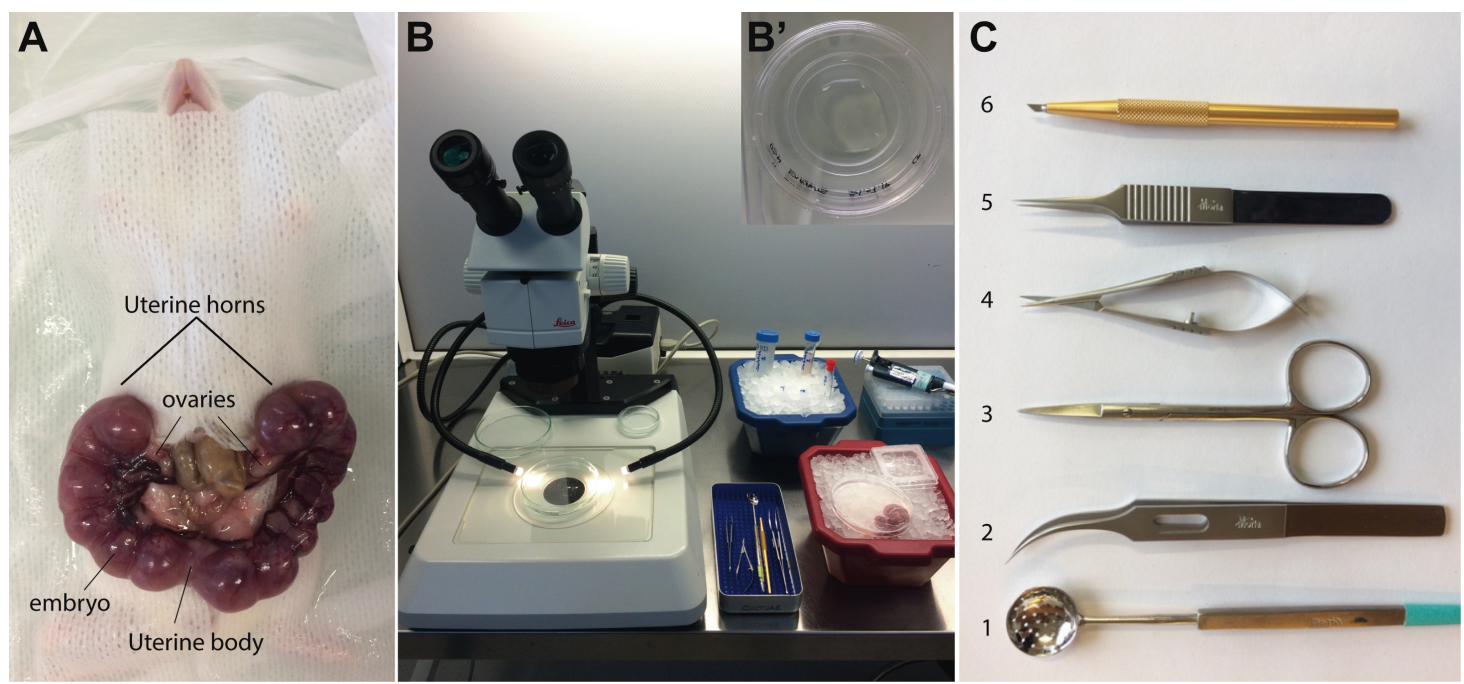

Figure 1. Presentation of equipment and tools necessary for embryo recovery and

dissection. A. Ventral view of an E13.5 pregnant mouse. The two uterine horns are removed from the mouse. On this picture, the left uterine horn contains 6 embryos and the right horn contains 8 embryos. B. Typical set-up with the Stereomicroscope under the hood, dissecting tools, pipettes and cones, and ice buckets to maintain solutions, dishes and embryos at $4{ }^{\circ} \mathrm{C}$. $\mathrm{B}^{\prime}$ shows a fifty $\mathrm{mm}$ diameter IBIDI video dish coated with PLL, in which the explants are cultured. C. Surgery tools. (C1) Perforated spoon (Moria MC17); (C2) curved Forceps Moria 9987; (C3) Moria 9600 Spring Scissors-Straight; (C4) Moria 4877A scissors; (C5) Moria MC40 Forceps; C6: Standard incision scalpel, $45^{\circ}$, Aluminum handle, $n^{\circ} 745$, FEATHER.

\section{Dissection}

a. The first step of the dissection aims at removing, from each embryo, a piece of cartilage covered by olfactory mucosa tissue, in a reproducible manner in terms of ventro-dorsal, antero-posterior and lateral boundaries. This piece of tissue will be subsequently called "Olfactory Piece 1" (OP1- Figure 2D). All the subsequent steps are done using the stereomicroscope under the hood, with forceps and microscissors. 

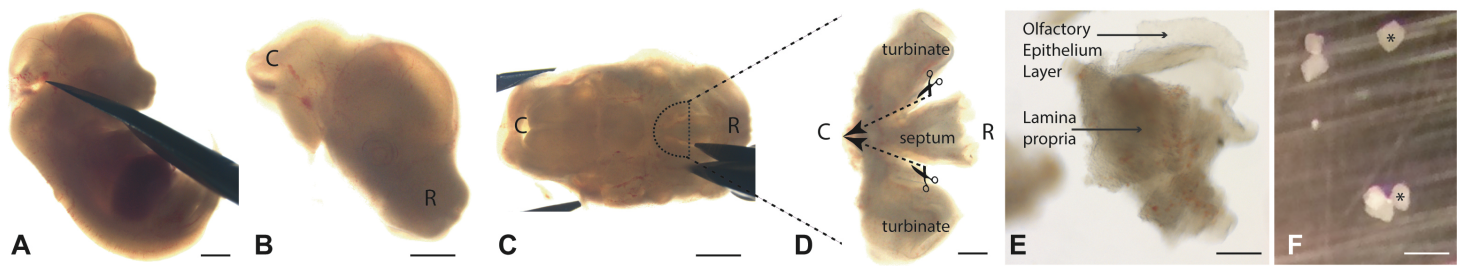

Figure 2. The main dissection steps for olfactory explant preparation. A. Cut the head using Moria 9600 Spring Scissors. Scale bar $=1 \mathrm{~mm}$. B. The head, see here on its right lateral side, is transferred into a clean dish. Scale bar $=1 \mathrm{~mm}$. C. Stabilize the head, ventral side up, with forceps (forceps tips are seen on the left), cut the hemi-palate with Moria 9600 Spring Scissors (right), to isolate OP1. Scale bar $=1 \mathrm{~mm}$. D. Detailed view of a dissected OP1. To further dissect OP1, separate the septum and the turbinals by cutting according to the dashed lines. Then, cut the septum in 4 pieces and the turbinals in 5 pieces. This gives OP2s. Scale bar $=200 \mu \mathrm{m}$. E. View of an OP2 during enzymatic treatment. The olfactory epithelium layer separates from the lamina propria. Scale bar $=200 \mu \mathrm{m}$. F. Explants with an asterisk are at the correct distance (about $1 \mathrm{~mm}$ ) to be cultured together in the same dish. The others are too close to each other and should be removed. R: Rostral; C: Caudal. Scale bar $=200 \mu \mathrm{m}$.

i. Isolate the upper part of the head by cutting through the mouth on both sides (Figures $2 \mathrm{~A}$ and $2 \mathrm{~B})$.

ii. Invert the head to see the under side (Figure 2C). At the E13.5 stage, the palate is not completely closed, and this orientation allows direct observation of the septum separating the right and left nasal cavities.

iii. Cut the lateral sides of the developing palate in the horizontal plane (Figure 2C). Once this is done, under the microscope it is possible to see the olfactory mucosa covering this cavity and attached to the septal, dorsal and lateral cartilages.

iv. Make a coronal section of the tissue through the septum and the turbinate bones (Figure 2C).

v. Detach the caudal part of OP1 from the olfactory bulb and cut beside the turbinates (Figure 2C, line).

vi. Extract OP1 (Figure 2D), and store in L-15 in a 4-well dish on ice.

vii. Repeat the same sequence of dissection steps with the other embryos in order to collect all OP1s pieces.

b. The second step of the dissection aims at extracting small fragments of the olfactory epithelium layer from OP1 pieces.

i. Transfer a few OP1s into a clean glass Petri dish containing cold L-15.

ii. Use forceps to maintain them immobile, and cut the described below pieces (hereafter referred to as OP2s) using the microscalpel (Figure 2D). 
iii. Make 4 OP2s with the septum part and 5 OP2s with the turbinates. In the turbinates, cut in the curved areas in order to obtain future explants as flat as possible (they will attach more easily to the glass surface).

iv. Collect all OP2s in one clean well containing L-15 on ice.

v. Prepare the enzymatic solution of Trypsin and Pancreatin by pooling an aliquot of 200 $\mu \mathrm{l} 0.25 \%$ Trypsin with an aliquot of $200 \mu \mathrm{l}$ of $4 \mathrm{x}$ USP Pancreatin (1:1).

vi. Remove the L-15 containing the OP2s and replace it with the enzymatic solution. Avoid bubbles. Incubate at room temperature (30 min. maximum) to allow the Olfactory Epithelium layer to separate from the lamina propria. Facilitate this separation by gently pipetting up and down with a 1,000 $\mu$ pipette/tip once or twice during the incubation. To preserve tissue quality, do not exceed $30 \mathrm{~min}$ incubation. The lamina propria appears as a white cloudy tissue while the olfactory epithelium as a translucent layer (Figure 2E).

vii. When the olfactory epithelium (OE) appears to progressively detach from the lamina propria, stop the enzymatic reaction by adding $45 \mu \mathrm{l}$ of $10 \%$ FBS (Working aliquot 1 ).

viii. Gently mix by pipetting up and down once. Incubate $5 \mathrm{~min}$ at room temperature and transfer on ice.

ix. Under the stereomicroscope, remove the enzymatic solution and wash 3 times with the L-15; 5\% FBS (Solution A).

$\mathrm{x}$. Incubate culture medium (Solution $\mathrm{B}$ ) at $37^{\circ} \mathrm{C}$ in a water bath.

xi. Transfer some OP1 into a glass Petri dish, gently isolate the OE tissue from the lamina propria and cut the OE sheets using the micro-scalpel into small pieces of about 50-150 $\mu \mathrm{m}$ diameter; these are the final explants (Figure 2F).

xii. Store explants in a clean well containing L-15; 5\% FBS on ice.

xiii. Once laminin coating is finished (after minimum $3 \mathrm{~h}$ at $37{ }^{\circ} \mathrm{C}, 5 \% \mathrm{CO}_{2}$ ), remove the dishes from the incubator.

xiv. Remove the laminin (using a pump aspirator or a pipette) and replace it directly by the appropriate volume of culture medium (6 $\mathrm{ml}$ in a $50 \mathrm{~mm}$ diameter dish).

10. Arrangement of explants in the dish

a. Collect 5 to 6 explants using a $10 \mu$ Pipette + tip and transfer them one by one directly to the appropriate position.

b. Place the tip as close as possible to the glass surface without touching it (contact would damage the coating). Explants should slowly descend by gravity.

c. Make sure there is at least $1 \mathrm{~mm}$ between 2 explants (see Figure $2 \mathrm{~F}$ for scale). We usually put 5 to 6 explants per dish, forming a cross in the center.

d. Once the explants have settled, carefully put the dishes back in the incubator at $37^{\circ} \mathrm{C}, 5 \%$ $\mathrm{CO}_{2}$.

B. Explant biotinylation and incubation with streptavidin beads 
Notes:

a. After 1.5 days in vitro (DIV), axons are long enough $(200-300 \mu \mathrm{m})$ to perform micromanipulation experiments to assess axon tension.

b. The protocol involves the biotinylation of the live explants (including their axons), allowing Streptavidin coated beads to attach to biotinylated axons.

1. Biotinylation

The reagent EZ-Link Sulfo-NHS-SS-Biotin is used at $200 \mu \mathrm{g} / \mathrm{ml}$ for $30 \mathrm{~min}$ at $37^{\circ} \mathrm{C}$ (with gentle shaking):

a. For 3 dishes, use 2 aliquots of $1 \mathrm{mg}$ of Sulfo-NHS-SS-Biotin.

b. Wait for equilibration at $4{ }^{\circ} \mathrm{C}$ and briefly centrifuge the tubes.

C. Resuspend each aliquot in $166 \mu \mathrm{l}$ DMEM/F-12 and pool (= $332 \mu \mathrm{l}$ at $6 \mathrm{mg} / \mathrm{ml})$.

d. Remove $3 \mathrm{ml}$ conditioned medium from the culture and store at $37^{\circ} \mathrm{C}$ (will be used for Step B2).

e. Take out $500 \mu \mathrm{l}$ conditioned medium of the culture, add $99 \mu \mathrm{l}$ of biotin solution and put back in the culture $(=200 \mu \mathrm{g} / \mathrm{ml}$ in $3 \mathrm{ml})$.

f. Incubate for $30 \mathrm{~min}$ at $37^{\circ} \mathrm{C}$ with gentle shaking.

2. Strepta-beads (ProActive ${ }^{\circledR}$ Microspheres, Streptavidin Polymeric: $3 \mu \mathrm{m}$ diameter, CP01N-1, Bangs Laboratories, Inc., Polysciences Europe $\mathrm{GmbH}$ )

The bead density can vary from one batch to another. Our batch had a concentration of $6.826 \mathrm{x}$ $10^{7}$ beads/ml. Using a $1 / 5,000$ dilution of these beads, we had a final concentration of $1.36 \mathrm{x}$ $10^{4}$ beads $/ \mathrm{ml}$ in each dish, which was to a satisfactory density.

a. First, prepare the Strepta-Beads solution:

Note: The 1/5,000 dilution of the beads is performed in 2 steps.

i. Vortex the beads.

ii. Predilute the beads at 1/100 in DMEM/F-12 (e.g., $2 \mu \mathrm{l}$ in $200 \mu \mathrm{l}$ DMEM/F-12).

iii. Make a $1 / 50$ dilution of the $1 / 100$ predilution.

iv. Vortex the beads $1 / 100$ and put $60 \mu \mathrm{l}$ of this predilution in the stored medium ( $3 \mathrm{ml}$ ).

b. Once biotinylation step is complete, wash each culture 3 times with warm $1 \times \mathrm{PBS}\left(-\mathrm{CaCl}_{2}\right.$, $-\mathrm{MgCl}_{2}$ ) and replace with "Strepta-Beads solution". Incubate for $30 \mathrm{~min}$ (minimum) at $37^{\circ} \mathrm{C}$, $5 \% \mathrm{CO}_{2}$.

C. During this incubation step, thaw 1 Working aliquot 3 (BSA10\% [v/v] in $2 \mathrm{ml} \mathrm{DMEM/F-12)}$ and prepare Solution C (BSA 2\% [v/v] $10 \mathrm{ml}$ DMEM/F-12).

d. Add $1 \mathrm{ml}$ of Solution $\mathrm{C}$ to the $3 \mathrm{ml}$ culture medium of each culture dish. Keep the dishes at $37^{\circ} \mathrm{C}, 5 \% \mathrm{CO}_{2}$, until use.

3. Quality control of the axon biotinylation:

In order to check that axons are properly biotinylated, and able to be bound by beads, a quality control can be performed. This quality control consists of detecting biotin using streptavidin, and to verify that beads can bind to axons. 
a. Wash once the cultures with $1 \times$ PBS.

b. Fix the culture for 15 min with 4\% PFA at RT.

c. Wash three times with $1 \times$ PBS.

d. Permeabilize 10 min at RT with 1x PBS; 0.2\% Triton.

e. Block for $1 \mathrm{~h}$ at RT in $1 x$ PBS; $5 \%$ NDS; $0.1 \%$ Triton.

f. Dilute rat $\alpha-N$ cam at $1 / 2,000$ in $1 \times$ PBS; $1 \%$ NDS; $0.1 \%$ Triton and incubate $\mathrm{O} / \mathrm{N}$ at $4{ }^{\circ} \mathrm{C}$.

\section{The next day:}

a. Wash three times with $1 \times$ PBS.

b. Dilute Donkey a-rat Cy3 at 1/1,000, Strepta-Fitc at 1/400 and Hoechst at $1 / 1,000$ in $1 \times$ PBS; 1\% NDS; 0.1\%Triton and incubate for $1 \mathrm{~h}$ at RT.

c. Wash three times with $1 \times$ PBS.

d. Mount the coverslip to the microscope slide with an appropriate mounting medium (e.g., Mowiol ${ }^{\circledR}$ 4-88).

e. Observe the results using a fluorescence microscope (Figure 3).

A

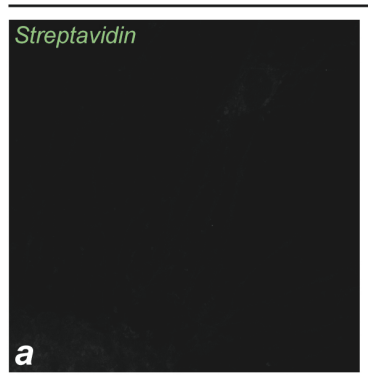

Control cultures

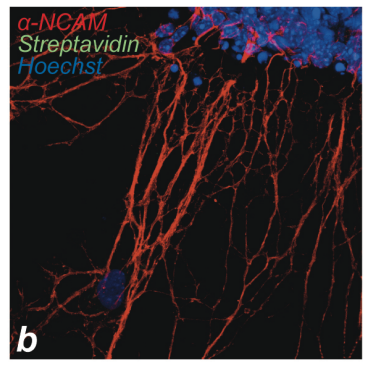

Biotinylated cultures

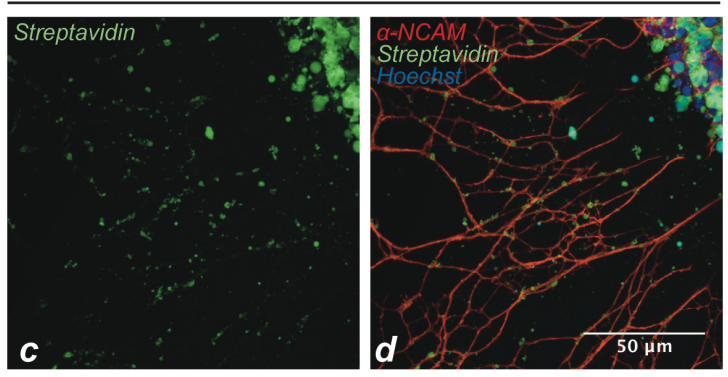

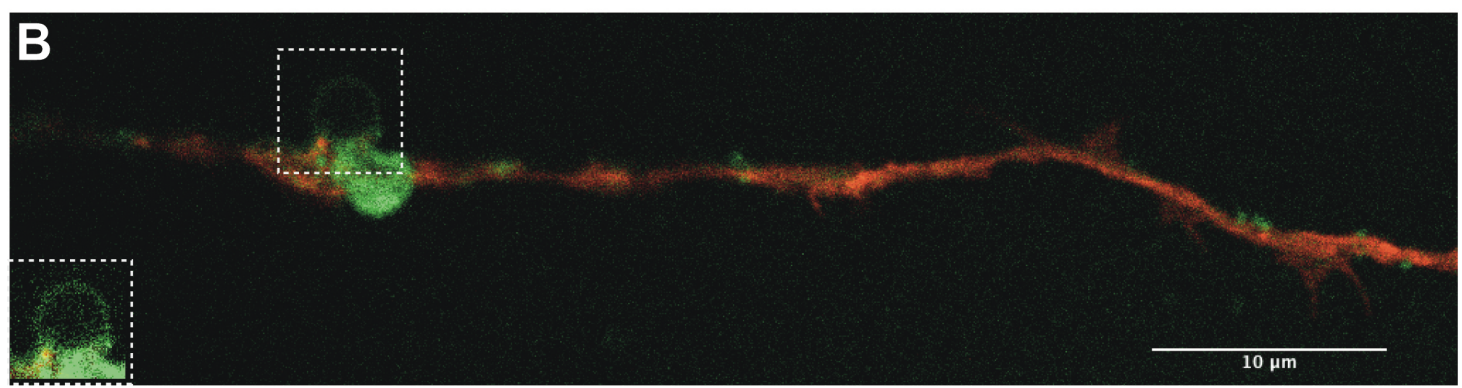

Figure 3. Quality control of explant biotinylation. A. Detection of biotin using Steptavidin-FITC on control explant cultures ( $A a, A b$, no biotin) or biotinylated cultures ( $A c, A d$ ). Explants were also treated with an $\alpha$-NCAM antibody detected with red fluorescence to label axons ( $\mathrm{A} b, \mathrm{~A} d$ ) and Hoescht to label cell nuclei (blue, $\mathrm{A} b, \mathrm{Ad}$ ). Aa and $\mathrm{A} c$ show the streptavidin labeling only, while $A b$ and $A d$ show the triple labeling. B. High magnification of a biotinylated axon, showing patches of biotinylation along the axon (green fluorescence), and a glass microbead (square) attached to a large patch. The bead is better seen in the inset.

C. Axon tension measurement using Biomembrane Force Probe 
The principle, tools and devices used in our experiments are described in details and illustrated by remarkable figures in Gourier et al., 2008. Therefore, in the protocol below, we will not describe the aspects that are already detailed in that technical publication. We will, however present, the detailed protocol and parameters used for tension measurement of olfactory axons.

1. Micropipette preparation

Micropipettes are prepared as described in Gourier et al. (2008), as follows:

a. Elongate a borosilicate glass capillary using the micropipette puller.

b. Using the microforge, as described in Gourier et al. (2008), adjust the micropipette diameter to give an aperture (inner diameter) of about $2 \mu \mathrm{m}$, to optimize the holding of the red blood cell by the pipette.

c. Fill the micropipette with culture medium.

d. Fix the micropipette to the micromanipulator mounted on the stage of the microscope and connected to the homemade manometers (Gourier et al., 2008).

e. Immerse the tip of the pipette in a drop of Solution $C$ deposited on a glass slide for $1 \mathrm{~h}$, allowing the saturation of the pipette glass with BSA.

2. Biomembrane force probe assembly

a. Remove the glass slide with the drop of BSA solution from the microscope stage.

b. Transport the dish containing the biotinylated explant cultures from the incubator to the microscope stage.

c. Measure the micropipette internal diameter, using the $40 x$ objective and the CCD camera.

d. Add the steptavidin-coated beads to the culture medium and allow them to settle for 3-5 min. Some of them will attach to axons.

e. Add biotinylated Red Blood Cells (RBC) to the culture medium.

f. Catch an RBC floating in the culture with the micropipette, via gentle aspiration $(\triangle P=$ 200-250 Pa).

g. Position this captured RBC in contact with a bead attached to an axon and wait at least 2 $\min$.

h. Check that the manipulated bead remains attached to both the axon and RBC, by slowly moving the pipette backwards and forwards. If the bead detaches from either the axon or the RBC, repeat the procedure with another bead attached to an axon.

i. In the favorable cases where the bead remains attached to both the axon and the RBC, proceed with the acquisition of data for tension measurement as follows:

i. The whole manipulation should be continuously recorded using the CCD camera.

ii. Pull the micropipette a short distance, induce elongation of the RBC; this often results in lateral deflection of the axon. An equilibrium is rapidly reached between the force induced by the probe and the transverse projection of the reaction force of the axon axial tension.

iii. Pull the micropipette again, a bit further, to induce an additional deformation of both the $\mathrm{RBC}$ and axon (a second equilibrium is reached). 
iv. Repeat gradual force application (through pulling) to acquire several equilibrium stages with different axon deflections, until the bead detaches from the RBC.

Note: Axons can be manipulated by pulling (as described above) and/or pushing, allowing the acquisition of more equilibrium stages. Detailed illustrations of olfactory axon tension measurement are provided in Šmít et al. (2017a and 2017b).

\section{Data analysis}

The video data obtained using this protocol allow the extraction of two key parameters necessary to calculate axon tension: 1) the deformation of the RBC (used here as a force transducer), allowing the calculation of the force (F) with which the bead (and hence the axon) is manipulated; and 2) the deflection of the axon, allowing the calculation of axon tension as a function of the applied force $F$. At equilibrium, the pulling force $F$ is balanced by the projection of the axon tension $(T)$ in the transverse direction, $2 T \sin \delta$, where $\delta$ is the angle of deflection of the axon (Šmít et al., 2017a and 2017b).

The procedures for assessing these two key parameters, as well as to calculate axon tension, are detailed in one software technical paper (Šmít et al., 2017b), and in a research paper (Šmít et al., 2017a), both in open access. Therefore, the data analyses will not be described further here.

\section{Notes}

1. Poly-L-Lysine was used in the experiments described in Šmít et al., 2017a and 2017b. Because Poly-D-Lysine (PDL) is thought to be less toxic for the cultures, we performed experiments using PDL $10 \mu \mathrm{g} / \mathrm{ml}$, and observed that it is suitable for explant adhesion to the coverslip.

2. PBS $1 \times 0.06 \%$ Glucose can be used for embryo dissection to reduce costs but L- 15 medium preserves viability and must be used at least for explant storage.

3. The microscalpel allows a nice cutting edge, preserves the integrity of the explant and reducing cell death. If this tool is not available, replace it with a $26 \mathrm{G}$ needle at the tip of a $1 \mathrm{ml}$ syringe.

4. The EZ-Link Sulfo-NHS-SS-Biotin is also available by weight $(100 \mathrm{mg})$. We find that using the No-Weigh Format enhances reproducibility.

5. DMEM/F-12 already contains $15 \mathrm{mM}$ HEPES, but if cell culture requires extended periods of manipulation outside of a $\mathrm{CO}_{2}$ incubator, add $10 \mathrm{mM}$ HEPES in the culture $1 \mathrm{~h}$ before processing.

6. To maintain the cultures at $37{ }^{\circ} \mathrm{C}$ during transport from the $\mathrm{CO}_{2}$ incubator to the micromanipulation laboratory, we stored the Petri dishes in a polystyrene box containing flasks of water preincubated at $37^{\circ} \mathrm{C}$.

7. Streptavidin-coated beads prepared as in (Gourier et al., 2008) can be replaced by a commercial version: ProActive ${ }^{\circledR}$ Microspheres, Streptavidin Polymeric: $3 \mu \mathrm{m}$ diameter, 
CP01N-1, Bangs Laboratories, Inc., Polysciences Europe GmbH. We have not tested these beads.

\section{$\underline{\text { Recipes }}$}

1. Working aliquot 1

FBS

a. Heat-inactivate the complement at $56{ }^{\circ} \mathrm{C}$ in a water bath for $30 \mathrm{~min}$

b. Prepare $3 \mathrm{ml}$ aliquots and store at $-20^{\circ} \mathrm{C}$

2. Working aliquot 2

$30 \%$ D-Glucose, prepare a $30 \%$ stock solution in $\mathrm{H}_{2} \mathrm{O}$ and store $1 \mathrm{ml}$ aliquots at $-20^{\circ} \mathrm{C}$

3. Working aliquot 3

BSA $10 \%$

DMEM/F-12

Note: Used for culture medium-solution 2 and for micropipettes.

a. Prepare a $10 \%$ BSA solution in DMEM/F-12, by dissolving $1 \mathrm{~g} \mathrm{BSA}$ in $9 \mathrm{ml}$ DMEM/F-12

b. Dissolution of BSA is enhanced by warming the DMEM/F-12 at $37^{\circ} \mathrm{C}$ and vortex

c. Store on ice and sterilize with a $0.2 \mu \mathrm{m}$ filter

d. Make $2 \mathrm{ml}$ aliquots and store at $-20^{\circ} \mathrm{C}$

Note: The following solutions have to be prepared on the day of the experiment (see Procedure).

4. Solution $A$

L-15 with 5\% FBS

a. In a $50 \mathrm{ml}$ sterile tube, prepare $47.5 \mathrm{ml} \mathrm{L}-15$ and add $2.5 \mathrm{ml} \mathrm{FBS} \mathrm{(working} \mathrm{aliquot} 1$ )

b. Sterilize with a $0.2 \mu \mathrm{m}$ filter and store on ice

5. Solution B (Culture medium)

DMEM/F12 containing 1\% N2 supplement, $0.1 \mathrm{mg} / \mathrm{ml}$ Gentamycin, 1.5\% D-Glucose, 1\% BSA and $7 \mu \mathrm{g} / \mathrm{ml}$ ascorbic acid

\section{Preparing $20 \mathrm{ml}$ of Solution B}

Six milliliters per $50 \mathrm{~mm}$ diameter dish is necessary for culture, e.g., for 3 dishes, in a sterile 50 $\mathrm{ml}$ tube, use an aliquot of all necessary components to prepare $20 \mathrm{ml}$ of Solution B:

a. Under sterile conditions, prepare $16.6 \mathrm{ml}$ of DMEM/F-12 in a $50 \mathrm{ml}$ tube and add $200 \mu \mathrm{l}$ of $\mathrm{N} 2$ supplement, $200 \mu \mathrm{l}$ Gentamycin, $1 \mathrm{ml}$ D-Glucose 30\% (working aliquot 2), $2 \mathrm{ml}$ of 10\% BSA; DMEM/F-12 (working aliquot 3) and $28 \mu \mathrm{l}$ ascorbic acid

b. Sterilize using a $0.2 \mu \mathrm{m}$ filter and store the culture medium on ice until the explants are ready

6. Solution C

2\% BSA in DMEM/F12 (for the micropipettes)

\section{Preparing $10 \mathrm{ml}$ Solution C}



a. Add $200 \mu \mathrm{g} \mathrm{BSA}$ into $10 \mathrm{ml}$ DMEM/F12 in a $15 \mathrm{ml}$ tube
b. Incubate at $37^{\circ} \mathrm{C}$ in a water bath until complete dissolution
c. Store on ice to equilibrate at $4{ }^{\circ} \mathrm{C}$
d. Sterilize using a $0.2 \mu \mathrm{m}$ filter and store on ice

7. Solution $\mathrm{D}$ (for red blood cell biotinylation)

$0.1 \mathrm{M}$ carbonate-bicarbonate, $\mathrm{pH} 8.5$

a. Prepare the two following solutions: D1: $0.1 \mathrm{M} \mathrm{NaHCO}_{3}: 4.2 \mathrm{~g} / 100 \mathrm{ml}$; D2: $0.1 \mathrm{M} \mathrm{Na}_{2} \mathrm{CO}_{3}$ : $1.06 \mathrm{~g} / 100 \mathrm{ml}$

b. Add solution $\mathrm{D} 1$ to solution $\mathrm{D} 2$ under $\mathrm{pH}$ monitoring, until $\mathrm{pH}$ reaches 8.5

8. Solution $\mathrm{E}$ (for red blood cell biotinylation)

$25 \mathrm{mM}$ Tris

$150 \mathrm{mM} \mathrm{NaCl}, \mathrm{pH} 7.5$

a. To $800 \mathrm{ml}$ of water, add $3 \mathrm{~g}$ of Tris base and $8.8 \mathrm{~g}$ of $\mathrm{NaCl}$

b. Adjust the $\mathrm{pH}$ to 7.5 using $1 \mathrm{~N} \mathrm{HCl}$, and add water q.s.p. $1000 \mathrm{ml}$

9. Solution $\mathrm{F}$ (for red blood cell biotinylation)

$0.01 \mathrm{M}$ phosphate buffer

$150 \mathrm{mM} \mathrm{NaCl}, \mathrm{pH} 7.4$

a. Prepare the following 4 solutions:

F1: $0.01 \mathrm{M} \mathrm{KH}_{2} \mathrm{PO}_{4}: 1.36 \mathrm{~g} / 100 \mathrm{ml}$

F2: $0.01 \mathrm{M} \mathrm{K}_{2} \mathrm{HPO}_{4}: 1.74 \mathrm{~g} / 100 \mathrm{ml}$

F3: $150 \mathrm{mM} \mathrm{NaCl}: 8.7 \mathrm{~g} / 100 \mathrm{ml}$

F4: Add solution $\mathrm{F} 1$ to solution $\mathrm{F} 2$ under $\mathrm{pH}$ monitoring, until $\mathrm{pH}$ reaches 7.4

b. Solution $\mathrm{F}$ is then obtained by adding $100 \mathrm{ml}$ solution F3 to $100 \mathrm{ml}$ solution F4 and add water q.s.p. $1,000 \mathrm{ml}$

10. Biotinylated Red Blood Cell (RBC) preparation (protocol from Gourier et al., 2008)

Note: All centrifugation steps of RBCs are performed for 2 min at 4,000 $\times$ g. All steps are performed at room temperature.

a. Collect 2-3 $\mu \mathrm{l}$ of human blood using a needle (TERUMO $26 \mathrm{G} 1 / 2$ ), and transfer to a $1.5 \mathrm{ml}$ Eppendorf tube

b. Wash 2 times in $1 \mathrm{ml}$ Solution $F$, each time by vortexing and subsequently pelleting by centrifugation (4,000 $\times \mathrm{g}, 2 \mathrm{~min})$, and removing the solution

c. Wash 3 times in Solution $D$ as above

d. Prepare $1 \mathrm{ml}$ solution of NHS-PEG 3400-biotin solution $(2 \mathrm{mg} / \mathrm{ml})$ in Solution D

e. Immediately mix $688.5 \mu \mathrm{l}$ of the above solution with the washed RBC, and 9,308.5 $\mu \mathrm{l}$ of Solution D

f. Incubate for $1 \mathrm{~h}$, under gentle agitation

g. Wash 3 times in Solution E

h. Suspend the pelleted RBCs in $10 \mathrm{ml}$ Solution $\mathrm{E}$

i. Incubate for $30 \mathrm{~min}$ under gentle agitation 
j. Wash 3 times in Solution $\mathrm{F}$

k. Suspend the pelleted RBC in $100 \mu$ l Solution F

I. The biotinylated RBC solution can be stored for 1 week at $4{ }^{\circ} \mathrm{C}$

\section{$\underline{\text { Acknowledgments }}$}

This work was supported by Sorbonne Université, CNRS, INSERM, ANR-2010-BLAN-1401-01 and NIH 5R01DC012441. We thank Christine Gourier and Pierre Soule for technical help and helpful discussion regarding BFP experiments, Ann Lohof for proofreading of the manuscript, and Daniel Šmít, Frederic Pincet and Martin Zapotocky for their scientific contribution to our overall project involving axon tension measurement.

\section{Competing interests}

The authors declare no conflicts of interest or competing interests.

\section{Ethics}

Our experiments on animals were approved by the Ile de France Ethics Committee (approval number 2018021915046521_v5).

\section{References}

1. Gangatharan, G., Schneider-Maunoury, S. and Breau, M. A. (2018). Role of mechanical cues in shaping neuronal morphology and connectivity. Biol Cell 110(6): 125-136.

2. Dennerll, T. J., Joshi, H. C., Steel, V. L., Buxbaum, R. E. and Heidemann, S. R. (1988). Tension and compression in the cytoskeleton of PC-12 neurites. II: Quantitative measurements. J Cell Biol 107(2): 665-674.

3. Dennerll, T. J., Lamoureux, P., Buxbaum, R. E. and Heidemann, S. R. (1989). The cytomechanics of axonal elongation and retraction. J Cell Biol 109(6 Pt 1): 3073-3083.

4. Evans, E., Ritchie, K. and Merkel, R. (1995). Sensitive force technique to probe molecular adhesion and structural linkages at biological interfaces. Biophys J 68(6): 2580-2587.

5. Franze, K., Janmey, P. A. and Guck, J. (2013). Mechanics in neuronal development and repair. Annu Rev Biomed Eng 15: 227-251.

6. Franze, K. (2013). The mechanical control of nervous system development. Development 140(15): 3069-3077.

7. Gourier, C., Jegou, A., Husson, J. and Pincet, F. (2008). A Nanospring named erythrocyte. The biomembrane force probe. In: Cellular and Molecular Bioengineering 1: 263-275. 
8. Rajagopalan, J., Tofangchi, A. and MT, A. S. (2010). Drosophila neurons actively regulate axonal tension in vivo. Biophys J 99(10): 3208-3215.

9. Šmít, D., Fouquet, C., Pincet, F., Zapotocky, M. and Trembleau, A. (2017a). Axon tension regulates fasciculation/defasciculation through the control of axon shaft zippering. Elife 6: e19907.

10. Šmít, D., Fouquet, C., Doulazmi, M., Pincet, F., Trembleau, A. and Zapotocky, M. (2017b). BFPTool: a software tool for analysis of Biomembrane Force Probe experiments. BMC Biophys 10: 2. 